\title{
Draft genomes of a male and female Australian jacky dragon
}

\section{(Amphibolurus muricatus)}

Ran $\operatorname{Tian}^{1,2}$, Han Guo ${ }^{1}$, Chen Yang ${ }^{1}$, Guangyi Fan $^{3,4}$, Sarah L. Whiteley ${ }^{5,6}$, Clare E. Holleley ${ }^{6}$, Inge Seim ${ }^{1,7}$, Arthur Georges ${ }^{5,8^{*}}$

${ }^{1}$ Integrative Biology Laboratory, College of Life Sciences, Nanjing Normal University, Nanjing 210046, China

${ }^{2}$ Jiangsu Key Laboratory for Biodiversity and Biotechnology, College of Life Sciences, Nanjing Normal University, Nanjing 210046, China

${ }^{3}$ BGI-Qingdao, Qingdao 266555, China

${ }^{4}$ BGI-Shenzhen, Shenzhen 518083, China

${ }^{5}$ Institute for Applied Ecology, University of Canberra, Canberra 2617, Australia

${ }^{6}$ Australian National Wildlife Collection, CSIRO, Canberra 2601, Australia

${ }^{7}$ School of Biology and Environmental Science, Queensland University of Technology, Brisbane 4000, Australia

${ }^{8}$ AusARG consortium, Bioplatforms Australia, Research Park Drive, Macquarie University, Macquarie Park 2109, Australia

*Corresponding author: Institute for Applied Ecology, University of Canberra, ACT 2601, Australia. E-mail: georges@aerg.canberra.edu.au 


\section{$1 \quad$ ABSTRACT}

2 Australia is remarkable for its lizard diversity, with very high endemicity because of

3 continental-scale diversification and adaptive radiation. We employed 10X Genomics

4 Chromium linked-reads technology to generate male and female draft genomes of the jacky

5 dragon (Amphibolurus muricatus), an Australian dragon lizard (family Agamidae). The

6 assemblies are $1.8 \mathrm{~Gb}$ in size and have a repeat content (38\%) and GC content (42\%) similar

7 to other dragon lizards. The contig N50 values for the assemblies were $37.2 \mathrm{~kb}$ (female) and

$828.8 \mathrm{~kb}$ (male), with corresponding scaffold N50 values of $720.5 \mathrm{~kb}$ and $369 \mathrm{~kb}$. The longest

9 scaffold was $6.5 \mathrm{Mb}$ in each assembly. The BUSCO completeness percentages were $92.2 \%$

10 and $90.8 \%$ respectively. These statistics are comparable to other lizard genomes assembled

11 using similar technology. Phylogenetic comparisons show that Australian dragon lizard

12 species split from a common ancestor around 33.4 million years ago. The $\operatorname{draft} A$. muricatus

13 assemblies will be a valuable resource for understanding lizard sex determination and the

14 evolution and conservation of Australian dragon lizards.

16 Keywords: agamid lizard; Agamidae; squamate; nuclear genome; genome assembly 


\section{Introduction}

The Australian jacky dragon, Amphibolurus muricatus (Figure 1), is lizard widespread in dry sclerophyll forests of south-eastern and eastern Australia (Cogger, 2014). It is a model species for biogeography (Pepper et al., 2014), evolutionary biology (Warner and Shine, 2008; Warner et al., 2013), social behaviour (Peters and Evans, 2003; Woo and Rieucau, 2013), and development (Esquerré et al., 2014; Whiteley et al., 2021).

Species in the genus Amphibolurus and Chlamydosaurus are a major clade in the Australian radiation of the Agamidae (Hugall et al., 2008). The draft assembly of $A$. muricatus, together with that of Pogona vitticeps (Georges et al., 2015), represents the first foray into generating the necessary high-quality genomes for the Agamidae. In particular, A.muricatus occupies mesic habitats and so is intermediate between the Australian water dragon Intellagama lesueurii and the forest dragon Lophosaurus boydii that occupy hydric habitats and the central bearded dragon Pogona vitticeps and the Lake Eyre dragon Ctenophorus maculosus, for example, that occupy more xeric habitats. As such, it is one of several species important for understanding genomic adaptation to the progressive aridity that has occurred in Australia in the past 15 Myr. Amphibolurus muricatus is also of particular interest because it has temperature-dependent sex determination (TSD) (Harlow and Taylor, 2000) and it is unclear as to whether this arises from classical TSD or a combination of genetic and environmental influences (Whiteley et al. 2021). Studies of the underlying mechanisms of TSD require a genome assembly and knowledge of genome organisation to identify genes on the sex chromosomes of species with genotypic sex determination (GSD) and their chromosomal and gene homology in closely related TSD species. This is particularly so in species with TSD that show evidence of cryptic residual or de novo genotypic influence on offspring sex ratios, as is suspected for A. muricatus (Whiteley et al., 2021).

Here, we employ high-throughput linked-read sequencing (10X Genomics) (Zheng et al., 2016) to generate draft, annotated genome assemblies for a male and a female $A$. muricatus that are comparable in contiguity and completeness to other published Agamid genomes generated using Illumina short-read technologies (Georges et al., 2015). We used transcriptomes sequenced and assembled for A. muricatus and published assemblies (Anolis, Varanus, Pogona) to annotate the genomes. Our assemblies will provide a resource to increase capacity and accelerate the progress of studies into the evolution, ecology, and conservation of Australian dragon lizards. 


\section{Materials and methods}

\section{Sample collection}

58 To reduce the high heterozygosity that presented difficulties in the assembly of the genome of

59 Pogona vitticeps (Georges et al., 2015), we generated inbred lines of $A$. muricatus. The

60 founding male and female pair were sourced from the wild and bred in captivity. The two

61 animals used to generate the genome were obtained from the fourth generation of the inbred

62 pedigree produced by sib-sib matings and back crossing (see Figure S1 for the complete

63 pedigree). The male (AA069033) and female (AA069032) individual used for the genome

64 and transcriptome sequencing were humanely euthanised via intraperitoneal injection of

65 sodium pentobarbitone $(60 \mathrm{mg} / \mathrm{ml}$ in isotonic saline). Organs were rapidly dissected and snap

66 frozen in liquid nitrogen.

\section{Linked-read whole-genome sequencing and de novo assembly}

69 High molecular weight DNA was extracted from liver (female) and blood (male) using the

70 Gentra Puregene DNA Isolation kit (Qiagen). DNA yield and quality was assessed using a

71 NanoDrop spectrophotometer (Thermo Fisher Scientific, Waltham, MA, USA) and a Qubit

72 fluorometer (Thermo Fisher Scientific). Male and female A. muricatus genome sequencing

73 libraries were constructed on the Chromium system (10X Genomics, Pleasanton, CA, USA)

74 by the Ramaciotti Centre for Genomics (Sydney, Australia). The Chromium instrument

75 enables unique barcoding of long stretches of DNA on gel beads. The barcodes allow later

76 reconstruction of long DNA fragments from a series of short DNA fragments with the same

77 barcode (i.e. linked-reads). After barcoding, DNA was sheared into smaller fragments and

78 sequenced on the NovaSeq 6000 platform (Illumina, CA, USA) to generate 151 bp paired-

79 end (PE) reads. A total of 904.9 M raw 10X Genomics Chromium linked-reads were

80 generated.

81 To estimate the genome sizes and heterozygosity of the female and male A. muricatus

82 samples, we used GenomeScope (Vurture et al., 2017). Briefly, we employed KmerGenie

83 v1.7051 (Chikhi and Medvedev, 2014) and short-read sequencing data (paired-ends reads

84 generated above) to determine the optimal $k$-mer-value range, indicating that a minimum $k$ -

85 mer length of 31 and 41 for the male and female, respectively. Next, $k$-mers in the paired-end

86 sequencing reads were counted and converted to histogram files using Jellyfish (v2.2.10)

87 (Marcais and Kingsford, 2011), followed by analysis using GenomeScope v1.0. 
Raw 10X data were assembled with Supernova v2.1.1 (Weisenfeld et al., 2017) and a FASTA file was generated using the 'pseudohap style' option in Supernova mkoutput. All female ( 450 M) and male ( 550 M) read pairs were utilised (female sequencing depth $c a$ $50.3 x$; male, $c a 47.8 x$ ). The resulting assembly was further scaffolded with ARKS v1.0.3 (Coombe et al., 2018), reusing the 10X reads, and the companion LINKS program (v1.8.7) (Warren et al., 2015). ARKS employs a $k$-mer approach to map linked barcodes to the contigs in the initial Supernova assembly to generate a scaffold graph with estimated distances for LINKS input. Next, RNA-seq reads (from brain, ovary, and testis; see below) were filtered (i.e. cleaned) to remove adapters and low-quality reads using Flexbar v3.4.0 and used to further re-scaffold the assembly with P_RNA_scaffolder (Zhu et al., 2018). The default Flexbar settings discards all reads with any uncalled bases. A final round of scaffolding was performed on the resulting assembly using L_RNA_scaffolder (Xue et al., 2013). We used GapCloser v1.12 (part of SOAPdenovo2) (Luo et al., 2012) to fill gaps in the assembly. GapCloser was run using the parameter -1 150) and cleaned 10X Genomics reads PE reads. Genome assemblies were assessed using BUSCO 5.0.0_cv1 (Seppey et al., 2019).

\section{RNA-seq and transcriptome assembly}

Raw data 125 bp PE reads, generated on an Illumina HiSeq 2500 instrument was filtered using Flexbar v3.4.0 (Dodt et al., 2012; Roehr et al., 2017) with default settings (removes reads with any uncalled bases). Any residual ribosomal RNA reads (the majority removed by poly(A) selection prior to sequencing library generation) were removed using SortMeRNA v2.1b (Kopylova et al., 2012) against the SILVA v119 ribosomal database (Quast et al., 2013). Tissue transcriptomes were de novo assembled using Trinity v2.11.0 (Grabherr et al., 2011; Haas et al., 2013; Henschel et al., 2012) and assessed using BUSCO 5.0.0_cv1 (Seppey et al., 2019).

\section{Genome annotation}

We identified repetitive elements by integrating homology and de novo prediction data. Protein-coding genes were annotated using homology-based prediction, de novo prediction, and RNA-seq-assisted prediction methods.

Homology-based transposable elements (TE) annotations were obtained by interrogating a genome assembly with known repeats in the Repbase database v16.02 (Bao et al., 2015) using RepeatMasker v4.0.5 (DNA-level) (Tarailo-Graovac and Chen, 2009) and RepeatProteinMask (protein-level; implemented in RepeatMasker). De novo TE predictions 
122 were obtained using RepeatModeler v1.1.0.4 (Smit and Hubley, 2010) and LTRharvest

123 v1.5.8 (Ellinghaus et al., 2008) to generate database for a RepeatMasker run. Tandem Repeat

124 Finder (v4.07) (Benson, 1999) was used to find tandem repeats (TRs) in the genome. A non-

125 redundant repeat annotation set was obtained by combining the above data.

126 Protein-coding genes were annotated using homology-based prediction, de novo

127 prediction, and RNA-seq-assisted [generated from ovary, testis, and brain (both sexes)]

128 prediction methods. Sequences of homologous proteins from three lizards [Anolis

129 carolinensis (green anole) assembly AnoCar2.0 (RefSeq assembly GCF_000090745.1)

130 (Alfoldi et al., 2011); Varanus komodoensis (Komodo dragon) assembly ASM479886v1

131 (GCA_004798865.1) (Lind et al., 2019); and Pogona vitticeps (central bearded dragon)

132 assembly pvi1.1 (GCF_900067755.1)] (Georges et al., 2015) were downloaded from NCBI.

133 These protein sequences were aligned to the repeat-masked genome using BLAT v0.36

134 (Kent, 2002). GeneWise v2.4.1 (Birney et al., 2004) was employed to generate gene

135 structures based on the alignments of proteins to a genome assembly. De novo gene

136 prediction was performed using AUGUSTUS v3.2.3 (Stanke et al., 2006), GENSCAN v1.0

137 (Burge and Karlin, 1997), and GlimmerHMM v3.0.1 (Majoros et al., 2004) with a human

138 training set. Transcriptome data (cleaned reads) were mapped to the assembled genome using

139 HISAT2 v2.1.0 (Kim et al., 2019) and SAMtools v1.9 (Li et al., 2009), and coding regions

140 were predicted using TransDecoder v5.5.0 (Grabherr et al., 2011; Haas et al., 2013). A final

141 non-redundant reference gene set was generated by merging the three annotated gene sets

142 using EvidenceModeler v1.1.1 (EVM) (Haas et al., 2008) and excluding EVM gene models

143 with only ab initio support. The gene models were translated into amino acid sequences and

144 used in local BLASTp (Camacho et al., 2009) searches against the public databases Kyoto

145 Encyclopedia of Genes and Genomes (KEGG; v89.1) (Kanehisa and Goto, 2000), NCBI non-

146 redundant protein sequences (NR; v20170924) (O'Leary et al., 2016), Swiss-Prot (release-

147 2018_07) (UniProt Consortium, 2012), TrEMBL (Translation of EMBL [nucleotide

148 sequences that are not in Swiss-Prot]; release-2018_07) (O'Donovan et al., 2002), and

149 InterPro (v69.0) (Mitchell et al., 2019).

\section{Phylogeny and divergence time estimation}

152 In addition to A. carolinensis, V. komodoensis, and $P$. vitticeps (see section above), the

153 genome and sequences of homologous proteins from Gekko japonicus (Schlegel's Japanese

154 gecko) assembly Gekko_japonicus_V1.1 (GCA_001447785.1) (Liu et al., 2015) and

155 Crotalus tigris (tiger rattlesnake) assembly ASM1654583v1 (GCA_016545835.1) (Margres 
156 et al., 2021) were downloaded from NCBI. The genome and annotations of Ophisaurus

157 gracilis (Anguidae lizard) were downloaded from GigaDB (Song et al., 2015a; Song et al.,

158 2015b). No gene annotation data was available for three species: Intellagama lesueurii

159 (Australian water dragon; assembly EWD_hifiasm_HiC generated as part of the AusARG

160 consortium and (downloaded from DNA Zoo (Cheng et al., 2021; Dudchenko et al., 2017;

161 Dudchenko et al., 2018)) and the Chinese agamid lizards Phrynocephalus przewalskii

162 (Przewalski's toadhead agama) (Gao et al., 2019) and Phrynocephalus vlangalii (Ching Hai

163 toadhead agama) (Gao et al., 2019) (CNGBdb accession no. CNP0000203). Their protein-

164 coding genes were annotated using homology-based prediction, de novo prediction, and

165 RNA-seq-assisted prediction methods (see genome annotation section above).

166 We identified 4,242 high-confidence 1:1 orthologs by interrogating the predicted

167 proteins from the gene models of ten species using SonicParanoid v1.3.0 (Cosentino and

168 Iwasaki, 2019). The corresponding coding sequences (CDS) for each species were aligned

169 using PRANK v100802 (Loytynoja and Goldman, 2005) and filtered by Gblocks v0.91b

170 (Talavera and Castresana, 2007) to identify conserved blocks (removing gaps, ambiguous

171 sites, and excluding alignments less than 300 bp in size), leaving 4,242 genes. Maximum-

172 likelihood (ML) phylogenetic trees were generated using RaxML v7.2.8 (Stamatakis, 2006)

173 and IQ-Tree v2.1.3 (Minh et al., 2020) with three CDS data sets: the whole coding sequence

174 (whole-CDS), first codon positions, and fourfold degenerate (4d) sites. Identical topologies

175 and similar support values were obtained (1,000 bootstrap iterations were performed). The

176 divergence time between species was estimated using MCMCTree [a Bayesian molecular

177 clock model implemented in PAML v4.7 (Yang, 2007)] with the JC69 nucleotide substitution

178 model, and the whole-CDS ML tree and concatenated whole-CDS supergenes as inputs. We

179 used 100,000 iterations after a burn-in of 10,000 iterations. MCMCTree calibration points

180 (million years ago; Mya) were obtained from TimeTree (Kumar et al., 2017): G. japonicus-P.

181 przewalskii (190-206 Mya), V. komodoensis-O. gracilis (121-143 Mya), V. komodoensis-C.

182 tigris (156-174 Mya), V. komodoensis-A. carolinensis (155-175 Mya), I. lesueurii-A.

183 carolinensis (139-166 Mya), I. lesueurii-P. przewalskii (73-93 Mya), I. lesueurii-A.

184 muricatus (25.5-42.4 Mya), P. vitticeps-A. muricatus (20.2-34.6 Mya). 


\section{Results and discussion}

\section{Draft genome assembly and comparisons with other squamates}

188 We used GenomeScope (Vurture et al., 2017) to obtain a $k$-mer-based estimate of genome

189 size and heterozygosity from paired-end sequence reads. The size of the A. muricatus genome

190 is estimated to be around $1.8 \mathrm{~Gb}$ (Figure S2). The genome-wide heterozygosity from our

191 inbred A. muricatus lines was estimated to range from $0.58 \%$ (female) to $0.78 \%$ (male)

192 (Figure S2), slightly lower than the central bearded dragon (Pogona vitticeps) (0.85\%)

193 (Georges et al., 2015).

10X Genomics sequencing data were assembled into contigs, then oriented and merged into scaffolds. Combining ARKS and P_RNA_scaffolder (employs RNA-seq reads from brain, ovary, and testis) (Table S1) and L_RNA_scaffolders (employs Trinity transcriptome assemblies) (Tables S2 and S3), the scaffold N50 size of the female $A$. muricatus (named AmuF1.1) assembly improved by $38.3 \%$ (from $371.5 \mathrm{~kb}$ to $720.5 \mathrm{~kb}$ ), while the BUSCO completeness percentage improved from $84.1 \%$ to $92.2 \%$ (Table S4). The male assembly (AmuM1.1) saw a similar improvement, with the scaffold N50 and BUSCO score increasing from $180.2 \mathrm{~kb}$ to $369.9 \mathrm{~kb}$ and from $78.9 \%$ to $90.8 \%$, respectively. The final assemblies had a total scaffold length (contain gaps) of $1.84 \mathrm{~Gb}$ (female) and $1.83 \mathrm{~Gb}$ (male) with a longest scaffold $c a 6.5 \mathrm{Mb}$ in each assembly (Table 1). The contig N50 values for the assemblies were $37.2 \mathrm{~kb}$ (female) and $28.8 \mathrm{~kb}$ (male). The scaffold and contig N50 values are similar to those of other squamate genome assemblies (Figure 2), with the exception of the chromosome-assigned assemblies of Australian water dragon (Intellagama lesueurii; scaffold N50 268.9 Mb), tiger rattlesnake (Crotalus tigris; scaffold N50 2.1 Mb) (Margres et al., 2021), green anole (Anolis carolinensis; scaffold N50 150.1 Mb), and Komodo dragon (Varanus komodoensis; scaffold N50 23.8 Mb) (Lind et al., 2019). The BUSCO metrics of the A. muricatus assemblies indicate that they compare well to other squamate assemblies,

211 including agamids from Australia [P. vitticeps (Georges et al., 2015) and I. lesueurii

212 (Australian water dragon)] and China (toad-headed agamas of genus Phrynocephalus sp.

213 (Gao et al., 2019)) (Figure 3) and Table S5).

\section{Genome annotation}

216 The A. muricatus assemblies are composed of 38\% repeat elements and have a GC content of 217 42\% (Tables S4 and S6), similar to that of P. vitticeps (Georges et al., 2015) - with LINEs

218 being the predominant subtype. Protein-coding genes were annotated by combining 
219 transcriptome evidence with homology-based (A. carolinensis, V. komodoensis, and $P$.

220 vitticeps) and de novo gene prediction methods. Gene statistics (Table S8) (see (Georges et

221 al., 2015)) and gene set BUSCO scores (Table S9) are comparable to other squamates. Using

222 ab inito, transcriptome, and homology-based prediction methods, we functionally annotated

$22318,197(85.0 \%)$ and 17,360 (88.0\%) protein-coding genes in the female and male assembly

224 (Tables S10 and S11) and recovered $94.7 \%$ and $93.1 \%$ of 3,354 vertebrate benchmarking

225 universal single-copy orthologs (BUSCOs) (Seppey et al., 2019), respectively.

\section{Phylogenetic relationships}

228 To construct a time-calibrated species tree (Figure 4), we identified 4,242 high-confidence

229 single-copy orthologs from the female A. muricatus assembly and nine other squamate

230 species. There are currently five agamid lizard genome assemblies: three Australian dragon

231 lizard assemblies (A. muricatus, P. vitticeps, and I. lesueurii) and two toad-headed agama

232 assemblies (genus Phrynocephalus) (Gao et al., 2019; Georges et al., 2015). Our analysis

233 shows that the five agamid species shared an ancestor about 85.7 Mya [81.3-88.2 Mya 95\%

234 credibility interval (CI)]. We estimate that the three Australian dragon lizard species split

235 from a common ancestor about 33.4 Mya (95\% CI 28.8-39.1), while the lineages leading to

236 A. muricatus and $P$. vitticeps diverged 23.6 Mya (95\% CI 19.4-28.2). These observations are

237 in agreement with an appraisal from a small set of mitochondrial and nuclear genes (Hugall et

238 al., 2008).

240 Conclusions and perspectives

241 In this study, we generated the first annotated genome assemblies of Amphibolurus

242 muricatus. Overall, the assemblies are similar in quality to a range of squamate genomes and

243 will be immediately useful for researchers. Nevertheless, it is appreciated that the assemblies

244 can be further improved. Such efforts will be particularly important for future studies on

245 squamate chromosome evolution and sex determination. Single-tube Long Fragment Read

246 (stLFR) sequencing (Fan et al., 2019; Wang et al., 2019) and re-scaffolding using SLR-

247 superscaffolder (Guo et al., 2021), and chromatin conformation capture (Hi-C) (Lieberman-

248 Aiden et al., 2009) sequencing to generate a chromosome-level assembly is planned by the

249 AusARG initiative of Bioplatforms Australia (https://bioplatforms.com). In conclusion, the $A$.

250 muricatus assemblies described here should prove valuable for the understanding of agamid

251 lizard evolution, ecology, and conservation. 
252

253

254

255

256

257

258

259

260

261

262

263

264

265

266

267

268

269

270

271

272

273

274

275

276

277

278

279

280

281

282

283

284

285

286

Data availability

A. muricatus raw genome and transcriptome reads have been deposited to the NCBI Short Read Database (BioProject ID: PRJNA767251). The male and female A. muricatus assemblies are available at Zenodo (Tian et al., 2021b). Gene annotation files and associated FASTA files for A. muricatus (assembly AmuF1.1), I. lesueurii, P. przewalskii, and $P$. vlangalii are available at Zenodo (Tian et al., 2021c). A. muricatus transcriptome assemblies are available at Zenodo (Tian et al., 2021a). Various scripts used for data processing and analyses are available on GitHub at https://github.com/sciseim/JackyDragon.

\section{Acknowledgements}

We thank Dr Wendy Ruscoe and Jacqui Richardson for their assistance in generating the inbred line of A. muricatus and for animal husbandry.

\section{Conflict of interest}

The authors declare there is no conflict of interest.

\section{Ethics Approvals}

All sampling and breeding experiments were conducted with approval of the Animal Ethics Committee of the University of Canberra and in accordance with their Standard Operating Procedures.

\section{Funding}

Financial support for this work was provided by an Australian Research Council Discovery Grant (DP170101147; to A.G. and C.E.H.), a specially-appointed Professor of Jiangsu Province grant (to I.S.), the Jiangsu Science and Technology Agency (to I.S.), and the Jiangsu Foreign Expert Bureau (to I.S.).

\section{Literature cited}

Alfoldi, J., Di Palma, F., Grabherr, M., Williams, C., Kong, L., Mauceli, E., Russell, P., Lowe, C.B., Glor, R.E., Jaffe, J.D., et al. (2011). The genome of the green anole lizard and a comparative analysis with birds and mammals. Nature 477, 587-591.

Bao, W., Kojima, K.K., and Kohany, O. (2015). Repbase Update, a database of repetitive elements in eukaryotic genomes. Mob DNA 6, 11.

Benson, G. (1999). Tandem repeats finder: a program to analyze DNA sequences. Nucleic Acids Res 27, 573-580. 
287 Birney, E., Clamp, M., and Durbin, R. (2004). GeneWise and Genomewise. Genome Res 14, 288 988-995.

289 Burge, C., and Karlin, S. (1997). Prediction of complete gene structures in human genomic 290 DNA. J Mol Biol 268, 78-94.

291 Camacho, C., Coulouris, G., Avagyan, V., Ma, N., Papadopoulos, J., Bealer, K., and Madden, 292 T.L. (2009). BLAST+: architecture and applications. BMC Bioinformatics 10, 421.

293 Cheng, H., Concepcion, G.T., Feng, X., Zhang, H., and Li, H. (2021). Haplotype-resolved de

294

295

296 novo assembly using phased assembly graphs with hifiasm. Nat Methods 18, 170-175. Chikhi, R., and Medvedev, P. (2014). Informed and automated k-mer size selection for genome assembly. Bioinformatics 30, 31-37.

Cogger, H. (2014). Reptiles and amphibians of Australia (CSIRO publishing).

Coombe, L., Zhang, J., Vandervalk, B.P., Chu, J., Jackman, S.D., Birol, I., and Warren, R.L. (2018). ARKS: chromosome-scale scaffolding of human genome drafts with linked read kmers. BMC Bioinformatics 19, 234.

Cosentino, S., and Iwasaki, W. (2019). SonicParanoid: fast, accurate and easy orthology inference. Bioinformatics 35, 149-151.

Dodt, M., Roehr, J.T., Ahmed, R., and Dieterich, C. (2012). FLEXBAR-Flexible Barcode and Adapter Processing for Next-Generation Sequencing Platforms. Biology (Basel) 1, 895905.

Dudchenko, O., Batra, S.S., Omer, A.D., Nyquist, S.K., Hoeger, M., Durand, N.C., Shamim, M.S., Machol, I., Lander, E.S., Aiden, A.P., et al. (2017). De novo assembly of the Aedes aegypti genome using Hi-C yields chromosome-length scaffolds. Science 356, 92-95. Dudchenko, O., Shamim, M.S., Batra, S.S., Durand, N.C., Musial, N.T., Mostofa, R., Pham, M., St Hilaire, B.G., Yao, W., and Stamenova, E. (2018). The Juicebox Assembly Tools module facilitates de novo assembly of mammalian genomes with chromosome-length scaffolds for under \$1000. BioRxiv, 254797.

Ellinghaus, D., Kurtz, S., and Willhoeft, U. (2008). LTRharvest, an efficient and flexible software for de novo detection of LTR retrotransposons. BMC Bioinformatics 9, 18.

Esquerré, D., Keogh, J.S., and Schwanz, L.E. (2014). Direct effects of incubation temperature on morphology, thermoregulatory behaviour and locomotor performance in jacky dragons (Amphibolurus muricatus). Journal of Thermal Biology 43, 33-39.

Fan, H., Wu, Q., Wei, F., Yang, F., Ng, B.L., and Hu, Y. (2019). Chromosome-level genome assembly for giant panda provides novel insights into Carnivora chromosome evolution. Genome Biol 20, 267.

Gao, W., Sun, Y.B., Zhou, W.W., Xiong, Z.J., Chen, L., Li, H., Fu, T.T., Xu, K., Xu, W., Ma, L., et al. (2019). Genomic and transcriptomic investigations of the evolutionary transition from oviparity to viviparity. Proc Natl Acad Sci U S A 116, 3646-3655.

Georges, A., Li, Q., Lian, J., O'Meally, D., Deakin, J., Wang, Z., Zhang, P., Fujita, M., Patel, H.R., Holleley, C.E., et al. (2015). High-coverage sequencing and annotated assembly of the genome of the Australian dragon lizard Pogona vitticeps. Gigascience 4, 45.

Grabherr, M.G., Haas, B.J., Yassour, M., Levin, J.Z., Thompson, D.A., Amit, I., Adiconis, X., Fan, L., Raychowdhury, R., Zeng, Q., et al. (2011). Full-length transcriptome assembly from RNA-Seq data without a reference genome. Nat Biotechnol 29, 644-652.

Guo, L., Xu, M., Wang, W., Gu, S., Zhao, X., Chen, F., Wang, O., Xu, X., Seim, I., Fan, G., et al. (2021). SLR-superscaffolder: a de novo scaffolding tool for synthetic long reads using a top-to-bottom scheme. BMC Bioinformatics 22, 158.

Haas, B.J., Papanicolaou, A., Yassour, M., Grabherr, M., Blood, P.D., Bowden, J., Couger, M.B., Eccles, D., Li, B., Lieber, M., et al. (2013). De novo transcript sequence reconstruction from RNA-seq using the Trinity platform for reference generation and analysis. Nat Protoc 8 , 1494-1512. 
337 Haas, B.J., Salzberg, S.L., Zhu, W., Pertea, M., Allen, J.E., Orvis, J., White, O., Buell, C.R., 338 and Wortman, J.R. (2008). Automated eukaryotic gene structure annotation using

339 EVidenceModeler and the Program to Assemble Spliced Alignments. Genome Biol 9, R7.

340 Harlow, P.S., and Taylor, J.E. (2000). Reproductive ecology of the jacky dragon

341 (Amphibolurus muricatus): an agamid lizard with temperature $\square$ dependent sex determination.

342 Austral Ecology 25, 640-652.

343 Henschel, R., Lieber, M., Wu, L.-S., Nista, P.M., Haas, B.J., and LeDuc, R.D. (2012). Trinity

344 RNA-Seq assembler performance optimization. Paper presented at: Proceedings of the 1st

345 Conference of the Extreme Science and Engineering Discovery Environment: Bridging from

346 the eXtreme to the campus and beyond (Chicag, IL, USA: Association for Computing

347 Machinery).

348 Hugall, A.F., Foster, R., Hutchinson, M., and Lee, M.S. (2008). Phylogeny of Australasian

349 agamid lizards based on nuclear and mitochondrial genes: implications for morphological

350 evolution and biogeography. Biological Journal of the Linnean Society 93, 343-358.

351 Kanehisa, M., and Goto, S. (2000). KEGG: Kyoto Encyclopedia of Genes and Genomes.

352 Nucleic Acids Res 28, 27-30.

353 Kent, W.J. (2002). BLAT--the BLAST-like alignment tool. Genome Res 12, 656-664.

354 Kim, D., Paggi, J.M., Park, C., Bennett, C., and Salzberg, S.L. (2019). Graph-based genome

355 alignment and genotyping with HISAT2 and HISAT-genotype. Nat Biotechnol 37, 907-915.

356 Kopylova, E., Noe, L., and Touzet, H. (2012). SortMeRNA: fast and accurate filtering of

357

358

359 ribosomal RNAs in metatranscriptomic data. Bioinformatics 28, 3211-3217.

Kumar, S., Stecher, G., Suleski, M., and Hedges, S.B. (2017). TimeTree: A Resource for Timelines, Timetrees, and Divergence Times. Mol Biol Evol 34, 1812-1819.

360 Li, H., Handsaker, B., Wysoker, A., Fennell, T., Ruan, J., Homer, N., Marth, G., Abecasis,

361 G., Durbin, R., and Genome Project Data Processing, S. (2009). The Sequence

362 Alignment/Map format and SAMtools. Bioinformatics 25, 2078-2079.

363 Lieberman-Aiden, E., van Berkum, N.L., Williams, L., Imakaev, M., Ragoczy, T., Telling,

364 A., Amit, I., Lajoie, B.R., Sabo, P.J., Dorschner, M.O., et al. (2009). Comprehensive

365 mapping of long-range interactions reveals folding principles of the human genome. Science

366 326, 289-293.

367 Lind, A.L., Lai, Y.Y.Y., Mostovoy, Y., Holloway, A.K., Iannucci, A., Mak, A.C.Y., Fondi,

368 M., Orlandini, V., Eckalbar, W.L., Milan, M., et al. (2019). Genome of the Komodo dragon

369 reveals adaptations in the cardiovascular and chemosensory systems of monitor lizards. Nat

370 Ecol Evol 3, 1241-1252.

371 Liu, Y., Zhou, Q., Wang, Y., Luo, L., Yang, J., Yang, L., Liu, M., Li, Y., Qian, T., Zheng, Y.,

372 et al. (2015). Gekko japonicus genome reveals evolution of adhesive toe pads and tail regeneration. Nat Commun 6, 10033.

Loytynoja, A., and Goldman, N. (2005). An algorithm for progressive multiple alignment of sequences with insertions. Proc Natl Acad Sci U S A 102, 10557-10562.

Luo, R., Liu, B., Xie, Y., Li, Z., Huang, W., Yuan, J., He, G., Chen, Y., Pan, Q., Liu, Y., et al. (2012). SOAPdenovo2: an empirically improved memory-efficient short-read de novo assembler. Gigascience 1, 18.

Majoros, W.H., Pertea, M., and Salzberg, S.L. (2004). TigrScan and GlimmerHMM: two open source ab initio eukaryotic gene-finders. Bioinformatics 20, 2878-2879.

Marcais, G., and Kingsford, C. (2011). A fast, lock-free approach for efficient parallel counting of occurrences of k-mers. Bioinformatics 27, 764-770.

Margres, M.J., Rautsaw, R.M., Strickland, J.L., Mason, A.J., Schramer, T.D., Hofmann, E.P., Stiers, E., Ellsworth, S.A., Nystrom, G.S., Hogan, M.P., et al. (2021). The Tiger Rattlesnake genome reveals a complex genotype underlying a simple venom phenotype. Proc Natl Acad

386 Sci U S A 118. 
387 Minh, B.Q., Schmidt, H.A., Chernomor, O., Schrempf, D., Woodhams, M.D., von Haeseler,

388 A., and Lanfear, R. (2020). IQ-TREE 2: New Models and Efficient Methods for Phylogenetic

389 Inference in the Genomic Era. Mol Biol Evol 37, 1530-1534.

390 Mitchell, A.L., Attwood, T.K., Babbitt, P.C., Blum, M., Bork, P., Bridge, A., Brown, S.D.,

391 Chang, H.Y., El-Gebali, S., Fraser, M.I., et al. (2019). InterPro in 2019: improving coverage,

392 classification and access to protein sequence annotations. Nucleic Acids Res 47, D351-D360.

393 O'Donovan, C., Martin, M.J., Gattiker, A., Gasteiger, E., Bairoch, A., and Apweiler, R.

394 (2002). High-quality protein knowledge resource: SWISS-PROT and TrEMBL. Brief

395 Bioinform 3, 275-284.

396 O'Leary, N.A., Wright, M.W., Brister, J.R., Ciufo, S., Haddad, D., McVeigh, R., Rajput, B.,

397 Robbertse, B., Smith-White, B., Ako-Adjei, D., et al. (2016). Reference sequence (RefSeq)

398 database at NCBI: current status, taxonomic expansion, and functional annotation. Nucleic

399 Acids Res 44, D733-745.

400 Pepper, M., Barquero, M.D., Whiting, M.J., and Keogh, J.S. (2014). A multi-locus molecular

401 phylogeny for Australia's iconic Jacky Dragon (Agamidae: Amphibolurus muricatus):

402 Phylogeographic structure along the Great Dividing Range of south-eastern Australia.

403 Molecular Phylogenetics and Evolution 71, 149-156.

404 Peters, R.A., and Evans, C.S. (2003). Introductory tail-flick of the Jacky dragon visual

405 display: signal efficacy depends upon duration. Journal of Experimental Biology 206, 4293-

4064307.

407 Quast, C., Pruesse, E., Yilmaz, P., Gerken, J., Schweer, T., Yarza, P., Peplies, J., and

408 Glockner, F.O. (2013). The SILVA ribosomal RNA gene database project: improved data

409 processing and web-based tools. Nucleic Acids Res 41, D590-596.

410 Roehr, J.T., Dieterich, C., and Reinert, K. (2017). Flexbar 3.0 - SIMD and multicore

411 parallelization. Bioinformatics 33, 2941-2942.

412 Seppey, M., Manni, M., and Zdobnov, E.M. (2019). BUSCO: Assessing Genome Assembly

413 and Annotation Completeness. Methods Mol Biol 1962, 227-245.

414 Smit, A.F., and Hubley, R. (2010). RepeatModeler Open-1.0. 2008-2015. Available at

415 http://www.repeatmasker.org.

416 Song, B., Cheng, S., Sun, Y., Zhong, X., Jin, J., Guan, R., Murphy, R., Che, J., Zhang, Y.,

417 and Liu, X. (2015a). Anguidae lizard (Ophisaurusgracilis) genome assembly data. Dryad

418 Digital Repository doi 10, 100119.

419 Song, B., Cheng, S., Sun, Y., Zhong, X., Jin, J., Guan, R., Murphy, R.W., Che, J., Zhang, Y.,

420 and Liu, X. (2015b). A genome draft of the legless anguid lizard, Ophisaurus gracilis.

421 Gigascience 4, 17.

422 Stamatakis, A. (2006). RAxML-VI-HPC: maximum likelihood-based phylogenetic analyses

423

424

425

426

427

428

429

430

431

432

433

434

435 with thousands of taxa and mixed models. Bioinformatics 22, 2688-2690.

Stanke, M., Keller, O., Gunduz, I., Hayes, A., Waack, S., and Morgenstern, B. (2006).

AUGUSTUS: ab initio prediction of alternative transcripts. Nucleic Acids Res 34, W435439.

Talavera, G., and Castresana, J. (2007). Improvement of phylogenies after removing divergent and ambiguously aligned blocks from protein sequence alignments. Syst Biol 56, 564-577.

Tarailo-Graovac, M., and Chen, N. (2009). Using RepeatMasker to identify repetitive elements in genomic sequences. Curr Protoc Bioinformatics Chapter 4, Unit 410.

Tian, R., Guo, H., Yang, C., Fan, G., Whiteley, S.L., Holleley, C.E., Seim, I., and Georges, A. (2021a). Assembled transcriptomes of ovary, testis, and brain (male and female) of Amphibolurus muricatus (jacky dragon) generated using Trinity v2.11.0, https://doi.org/10.5281/zenodo.5523684 (Zenodo). 
436 Tian, R., Guo, H., Yang, C., Fan, G., Whiteley, S.L., Holleley, C.E., Seim, I., and Georges,

437 A. (2021b). Draft de novo genome assemblies of a male and female Amphibolurus muricatus

438 (jacky dragon), https://doi.org/10.5281/zenodo.5523788 (Zenodo).

439 Tian, R., Guo, H., Yang, C., Fan, G., Whiteley, S.L., Holleley, C.E., Seim, I., and Georges,

440 A. (2021c). Gene annotations of Amphibolurus muricatus (jacky dragon), Intellagama

441 lesueurii (Australian water dragon), Phrynocephalus przewalskii (Przewalski's toadhead

442 agama), and Phrynocephalus vlangalii (Ching Hai toadhead agama),

443 https://doi.org/10.5281/zenodo.5523656 (Zenodo).

444 UniProt Consortium (2012). Reorganizing the protein space at the Universal Protein

445 Resource (UniProt). Nucleic Acids Res 40, D71-75.

446 Vurture, G.W., Sedlazeck, F.J., Nattestad, M., Underwood, C.J., Fang, H., Gurtowski, J., and

447 Schatz, M.C. (2017). GenomeScope: fast reference-free genome profiling from short reads.

448 Bioinformatics 33, 2202-2204.

449 Wang, O., Chin, R., Cheng, X., Wu, M.K.Y., Mao, Q., Tang, J., Sun, Y., Anderson, E., Lam,

450 H.K., Chen, D., et al. (2019). Efficient and unique cobarcoding of second-generation

451 sequencing reads from long DNA molecules enabling cost-effective and accurate sequencing,

452 haplotyping, and de novo assembly. Genome Res 29, 798-808.

453 Warner, D., and Shine, R. (2008). The adaptive significance of temperature-dependent sex

454 determination in a reptile. Nature 451, 566-568.

455 Warner, D.A., Uller, T., and Shine, R. (2013). Transgenerational sex determination: the

456 embryonic environment experienced by a male affects offspring sex ratio. Scientific reports

$457 \quad 3,1-4$.

458 Warren, R.L., Yang, C., Vandervalk, B.P., Behsaz, B., Lagman, A., Jones, S.J., and Birol, I.

459 (2015). LINKS: Scalable, alignment-free scaffolding of draft genomes with long reads.

460 Gigascience 4, 35.

461 Weisenfeld, N.I., Kumar, V., Shah, P., Church, D.M., and Jaffe, D.B. (2017). Direct

462 determination of diploid genome sequences. Genome Res 27, 757-767.

463 Whiteley, S.L., Georges, A., Weisbecker, V., Schwanz, L.E., and Holleley, C.E. (2021).

464 Ovotestes suggest cryptic genetic influence in a reptile model for temperature-dependent sex

465 determination. Proceedings of the Royal Society B 288, 20202819.

466 Woo, K., and Rieucau, G. (2013). Efficiency of aggressive and submissive visual displays

467 against environmental motion noise in Jacky dragon (Amphibolurus muricatus). Ethology

468 Ecology \& Evolution 25, 82-94.

469 Xue, W., Li, J.T., Zhu, Y.P., Hou, G.Y., Kong, X.F., Kuang, Y.Y., and Sun, X.W. (2013).

470 L_RNA_scaffolder: scaffolding genomes with transcripts. BMC Genomics 14, 604 .

471 Yang, Z. (2007). PAML 4: phylogenetic analysis by maximum likelihood. Mol Biol Evol 24,

472 1586-1591.

473 Zheng, G.X., Lau, B.T., Schnall-Levin, M., Jarosz, M., Bell, J.M., Hindson, C.M.,

474 Kyriazopoulou-Panagiotopoulou, S., Masquelier, D.A., Merrill, L., Terry, J.M., et al. (2016).

475 Haplotyping germline and cancer genomes with high-throughput linked-read sequencing. Nat

476 Biotechnol 34, 303-311.

477 Zhu, B.H., Xiao, J., Xue, W., Xu, G.C., Sun, M.Y., and Li, J.T. (2018). P_RNA_scaffolder: a

478 fast and accurate genome scaffolder using paired-end RNA-sequencing reads. BMC

479 Genomics 19, 175.

480 
bioRxiv preprint doi: https://doi.org/10.1101/2021.10.11.463868; this version posted October 11, 2021. The copyright holder for this preprint (which was not certified by peer review) is the author/funder, who has granted bioRxiv a license to display the preprint in perpetuity. It is made available under aCC-BY-NC-ND 4.0 International license.

\section{Figure legends}

483

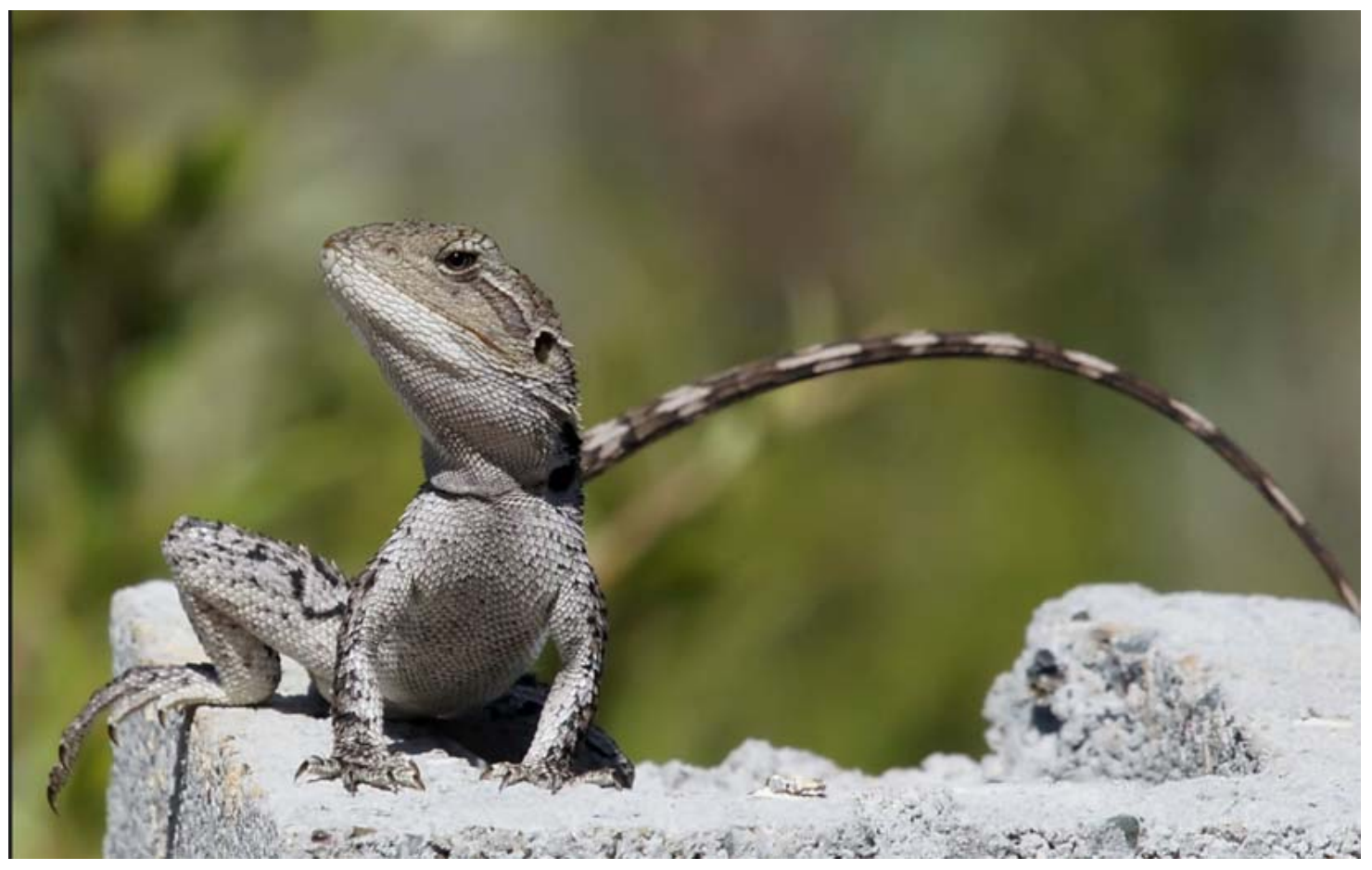

484 Figure 1 Photograph of an adult male jacky dragon (Amphibolurus muricatus). Image credit:

485 David Cook Wildlife Photography.

486 

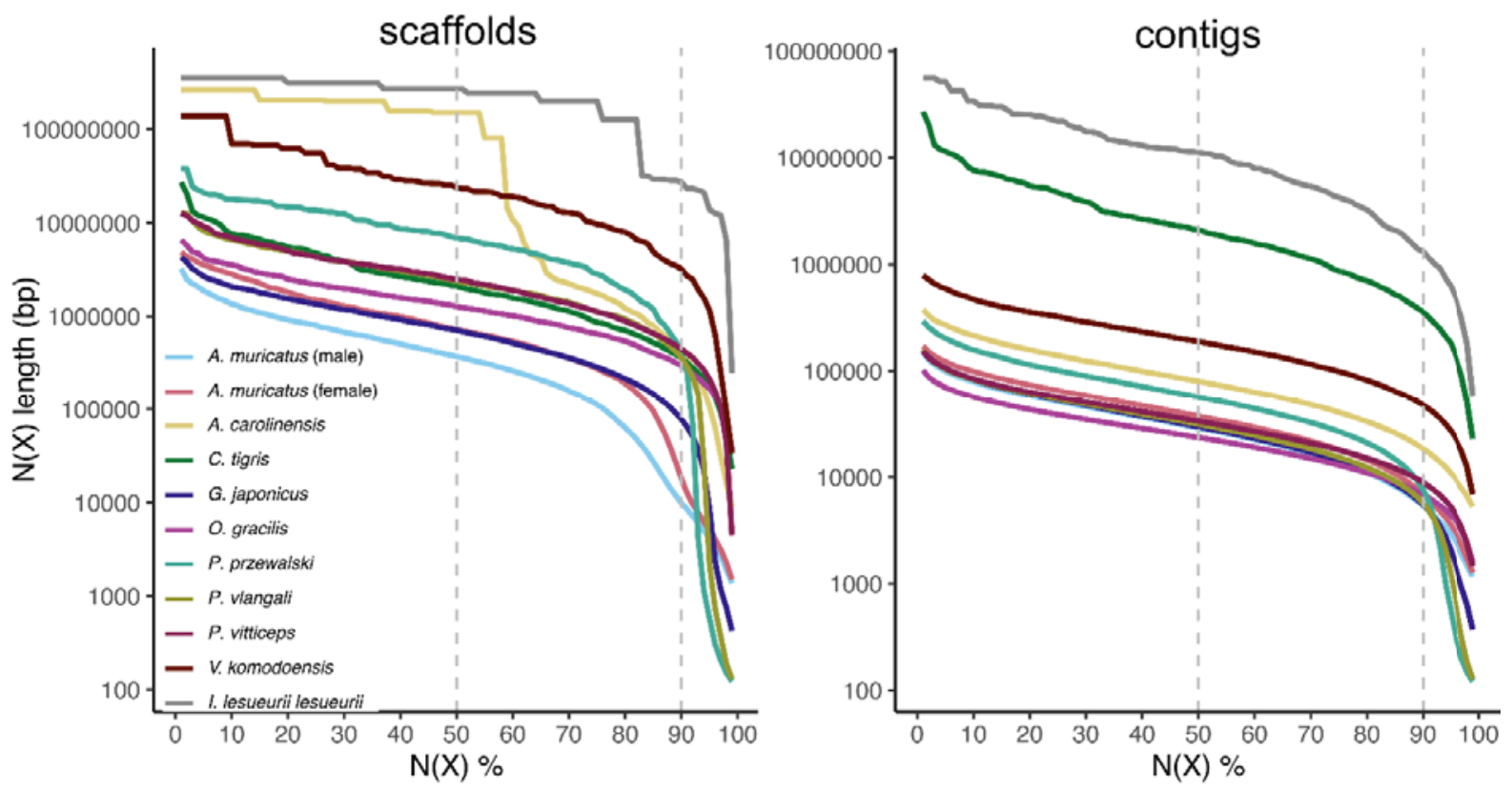

489 Figure 2 Comparison of the contiguity of two A. muricatus assemblies and nine publicly

490 available squamate assemblies. $\mathrm{N}(\mathrm{x}) \%$ graphs show the (A) contig and (B) scaffold lengths

491 ( $y$-axis), where $\mathrm{x} \%$ ( $x$-axis) of the genome assembly consist of scaffolds and contigs of at

492 least that size. Dashed, grey lines denote N50 and N90 values. 
bioRxiv preprint doi: https://doi.org/10.1101/2021.10.11.463868; this version posted October 11, 2021. The copyright holder for this preprint (which was not certified by peer review) is the author/funder, who has granted bioRxiv a license to display the preprint in perpetuity. It is made available under aCC-BY-NC-ND 4.0 International license.

494

495

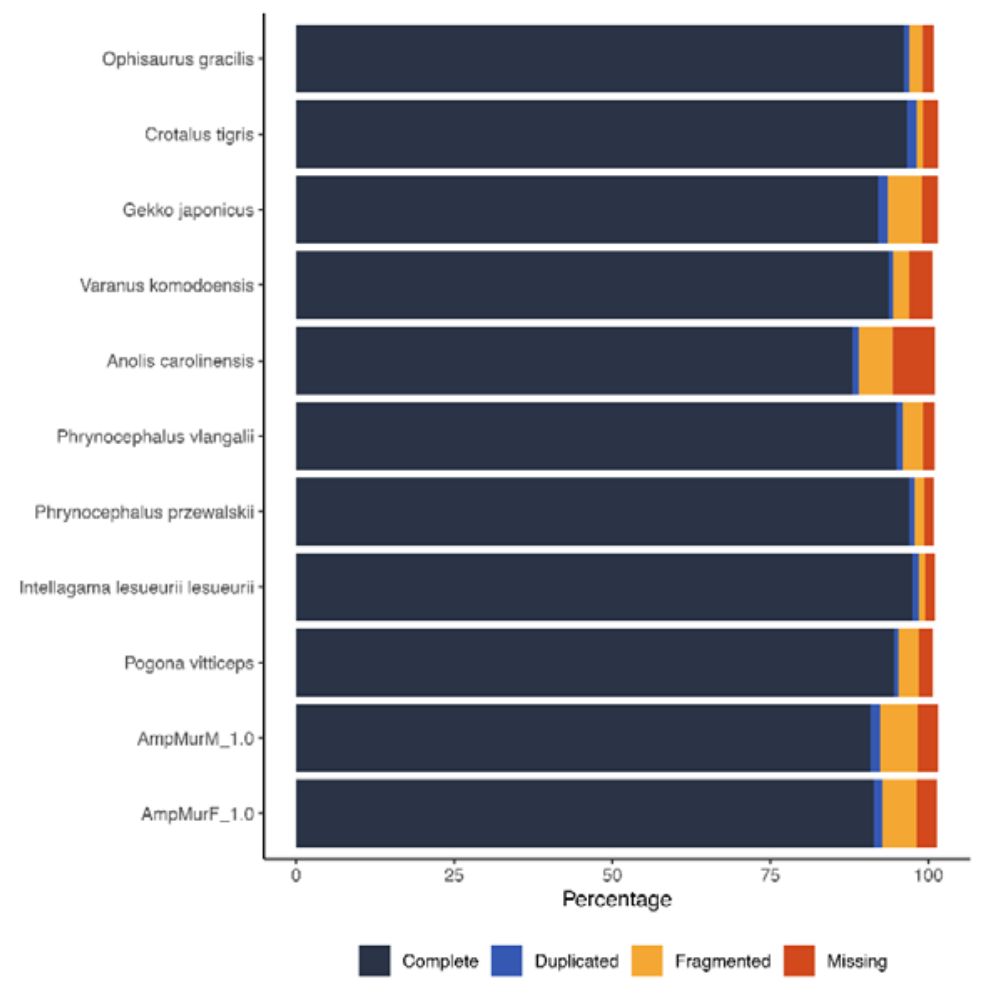

496 Figure 3 BUSCO assessment of assemblies from ten squamate species. All genome

497 assemblies were examined using the same version and library of BUSCO (5.0.0_cv1 with the

498 3,354-gene vertebrata_odb10 dataset). AmuF_1.1 and AmuM_1.1 denotes the female and

499 male A. muricatus assembly, respectively. 


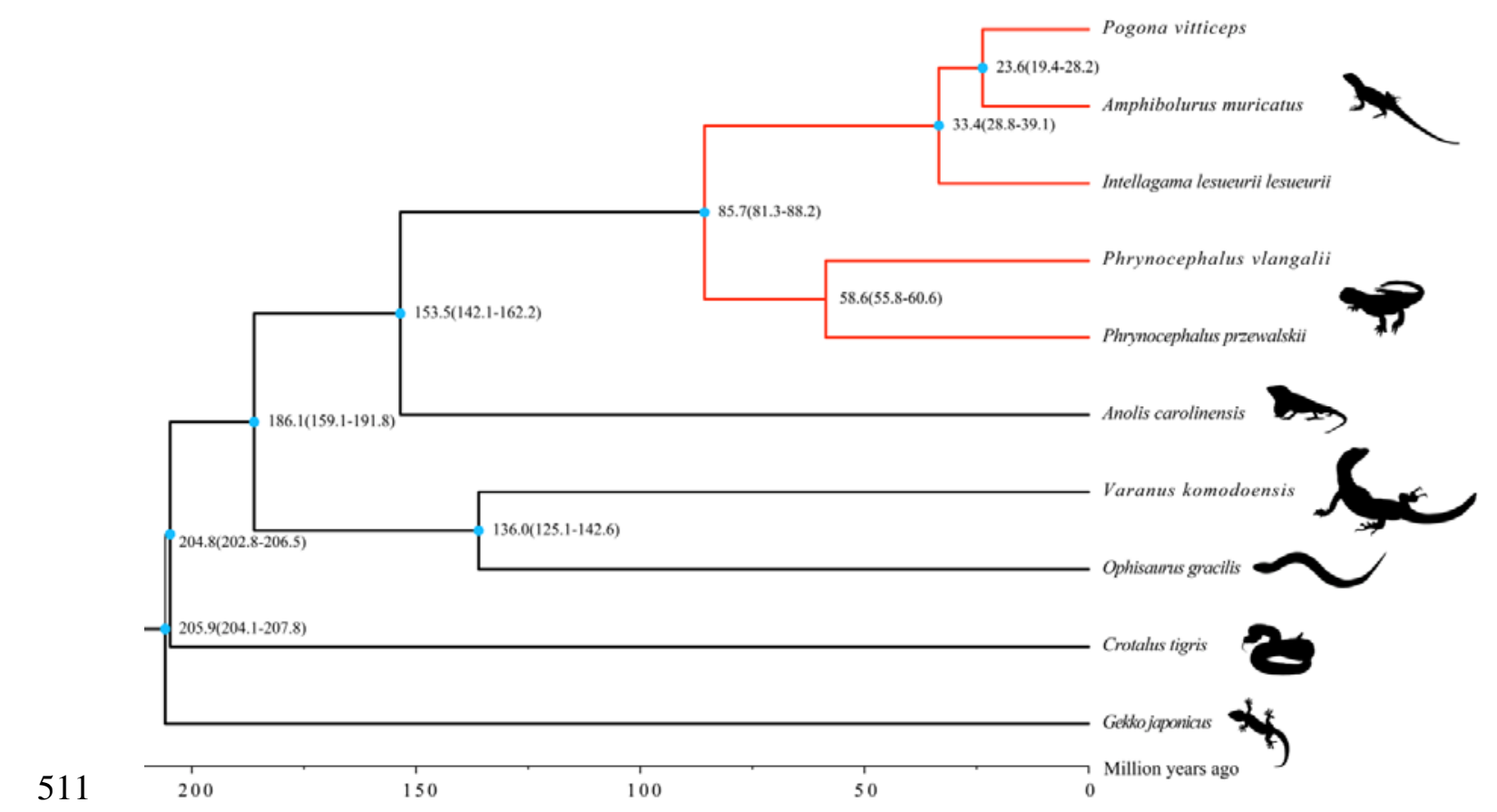

512 Figure 4 Inferred phylogeny of ten squamate species based on whole-coding sequences of

\section{4,242 1:1 orthologs. Numbers at nodes represent the estimated divergence time from present}

514 (million years ago; Mya) between lineages. Agamid (family Agamidae) lineages are indicated 515 in red.

516

517

518 
bioRxiv preprint doi: https://doi.org/10.1101/2021.1011.463868; this version posted October 11, 2021. The copyright holder for this preprint (which was not certified by peer review) is the author/funder, who has granted bioRxiv a license to display the preprint in perpetuity. It is made available under aCC-BY-NC-ND 4.0 International license.

\section{Tables}

520 Table 1 A. muricatus genome assembly statistics. Lengths in base pairs (bp).

\begin{tabular}{lll}
\hline Assembly methods & Female (AmuF.1.1) & Male (AmuM.1.1) \\
\hline Contig number & 124,200 & 151,787 \\
Contig length & $1,750,545,991$ & $1,741,048,453$ \\
Contig N50 (bp) & 37,220 & 28,761 \\
Contig max length & 348,284 & 288,200 \\
Scaffold number & 57,227 & 73,856 \\
Scaffold length & $1,841,491,868$ & $1,833,283,242$ \\
Scaffold N50 (bp) & 720,518 & 369,860 \\
Scaffold max length & $6,534,950$ & $6,446,322$ \\
Gaps (bp) & $90,945,877$ & $92,234,789$ \\
Gaps (\%) & 4.94 & 5.03 \\
GC content (\%) & 41.77 & 41.70 \\
\hline
\end{tabular}

521 